\title{
UNA NUEVA ESPECIE DE HOPLOPLEURA (PHTHIRAPTERA, ANOPLURA) PARÁSITA DE TRES ESPECIES DE BIBIMYS (MURIDAE, SIGMODONTINAE, RODENTIA)
}

\author{
Dolores del Carmen Castro ${ }^{1}$ \\ Alda Gonzalez ${ }^{2}$
}

\begin{abstract}
A NEW SPECIES OF HOPLOPLEURA (PHTHIRAPTERA, ANOPLURA) PARASITIC ON THREE SPECIES OF BIBIMYS (MURIDAE, SIGMODONTINAE, RODENTIA). A new species Hoplopleura massoiai is described, based on specimens collected on three species of Bibimys Massoia, 1979: B. torresi Massoia, 1979, B. chacoensis (Shamel, 1931) and B. labiosus (Winge, 1887), from Chaco, Misiones and Buenos Aires provinces, Argentina. Male, female, first nymphal instar, external architecture of eggs and sites of oviposition are described, providing differences with its close relative Hoplopleura scapteromydis Ronderos, 1965; comments on distribution of the new species on its hosts are done.
\end{abstract}

KEYWORDS. Phthiraptera, Anoplura, Hoplopleura, Rodentia, Argentina.

\section{INTRODUCCIÓN}

Una nueva especie de Hoplopleura Enderlein, 1904 es descripta de ejemplares obtenidos sobre Bibimys torresi Massoia, 1979, B. chacoensis (Shamel, 1931) y B. labiosus (Winge, 1887) de las provincias de Chaco, Misiones y Buenos Aires, Argentina. Esta nueva especie integra con Hoplopleura scapteromydis Ronderos, 1965 el grupo aitkeni del género Hoplopleura (JoHnson, 1972), grupo que cuenta ahora con 12 especies conocidas para Argentina (CASTRO et al., 1995, 1996). Se detallan los patrones de comportamiento oviposicional y se realizan comentarios a cerca de esta nueva especie, en las hasta hoy, tres especies conocidas del género Bibimys Massoia, 1979, analizándose la distribución geográfica de las especies del referido género de Sigmodontinae para Argentina.

1. Facultad de Ciencias Naturales y Museo, Paseo del Bosque, s/n, 1900, La Plata, Buenos Aires, Argentina. CONICET. (castrodreon@lpsat.com) 2. CEPAVE, calle 2, $\mathrm{n}^{\circ} 584,1900$, La Plata, Buenos Aires, Argentina. 


\section{MATERIAL Y MÉTODOS}

Los ejemplares estudiados fueron obtenidos directamente sobre sus hospederos y montados en preparaciones microscópicas convencionales siguiendo CASTRO \& Cicchino (1978). Para el estudio de las características coriónicas externas de los huevos, éstos fueron fijados en glutaraldehído al 10\%, y posteriormente procesados, acondicionados para su estudio, fotografiados y medidos con el microscopio electrónico de barrido. La tipificación nomenclatorial de las estructuras es según CASTRO et al. (1991). Las medidas de las características ultraestructurales se expresan en micrómetros. Las medidas corporales fueron tomadas directamente sobre los ejemplares aclarados y montados por medio de un ocular micrométrico y se representan en milímetros. Para la quetotaxia cefálica se sigue la nomenclatura de Kim \& Ludwig (1978) y para la referencia de los hospederos, RedFord \& Eisenberg (1992).

El material estudiado se halla depositado en las colecciones del Museo de La Plata (MLP).

\section{Hoplopleura massoiai sp. nov.}

(Figs. 1-10)

Holotipo macho (fig. 1). Cabeza apenas más larga que ancha, con su borde anterior rectilíneo y bordes laterales convexos; ángulos postantenales redondeados; quetotaxia cefálica: seta dorsal principal, larga y robusta; dorsal accesoria, pequeña y delgada; apical, cuatro delgadas; central anterior, una pequeña y delgada; ventral principal, una larga y robusta; ventral preantenal, una delgada y corta. Tórax mas ancho que largo, seta torácica esternal larga y delgada, placa torácica esternal más larga que ancha, llegando su porción posterior a contactar con la coxa III (fig. 4). Abdomen con placas esternales bien desarrolladas con setas uniformes en toda la extensión abdominal. Placas tergales bien desarrolladas a partir del tergito V, setación del tergito I-III (4, muy delgadas y de igual longitud) tergito IV-VIII (10-12, todas de iguales características ). Placas paratergales: II con dos lóbulos aguzados desiguales, siendo el ventral más largo que el dorsal, presentando en la escotadura dos setas de distinto tamaño; III con dos lóbulos aguzados pequeños y subiguales, escamación apenas marcada en su porción terminal, portando dos setas de distinto tamaño, siendo la dorsal mucho más larga y robusta que la ventral; IV-VI con dos lóbulos anchos terminando en ambos extremos en un lóbulo aguzado con una marcada escamación terminal y portando dos setas diminutas; VII, apenas insinuado el lóbulo dorsal, con dos macroquetas; VIII sin lóbulos, con dos macroquetas (fig. 1). Genitalia: placa basal bien desarrollada, larga y angosta, parámeros de mediano desarrollo

Tabla I. Dimensiones corporales de los adultos y ninfa I de Hoplopleura massoiai sp. nov., expresadas en milímetros. Las mismas constan del rango (R), media (X) y el desvío standard (SD); (HL, largo de la cabeza; HW, ancho máximo de la misma; THL, largo del tórax; THW, ancho máximo del mismo; AL, largo del abdomen; AW, ancho máximo del mismo; TL, largo total del cuerpo).

\begin{tabular}{|c|c|c|c|c|c|c|c|c|c|}
\hline & \multicolumn{3}{|c|}{$\begin{array}{c}\text { machos } \\
\mathrm{n}=3\end{array}$} & \multicolumn{3}{|c|}{$\begin{array}{c}\text { hembras } \\
\mathrm{n}=4\end{array}$} & \multicolumn{3}{|c|}{$\begin{array}{c}\text { ninfas } \\
\mathrm{n}=2\end{array}$} \\
\hline & $X$ & SD & $\mathrm{R}$ & $X$ & SD & $\mathrm{R}$ & $X$ & SD & $\mathrm{R}$ \\
\hline HL & 0,129 & 0,017 & $0,110-0,140$ & 0,119 & 0,003 & $0,114-0,122$ & 0,052 & 0,003 & $0,050-0,055$ \\
\hline HW & 0,171 & 0,010 & $0,164-0,184$ & 0,174 & 0,009 & $0,167-0,187$ & 0,159 & 0,002 & $0,158-0,161$ \\
\hline THL & 0,063 & 0,035 & $0,030-0,100$ & 0,116 & 0,004 & $0,111-0,121$ & 0,025 & 0,001 & $0,024-0,026$ \\
\hline THW & 0,262 & 0,010 & $0,250-0,269$ & 0,265 & 0,018 & $0,240-0,283$ & 0,225 & 0,002 & $0,223-0,227$ \\
\hline $\mathrm{AL}$ & 0,803 & 0,005 & $0,800-0,810$ & 0,891 & 0,063 & $0,829-0,948$ & 0,402 & 0,001 & $0,401-0,403$ \\
\hline AW & 0,596 & 0,006 & $0,590-0,600$ & 0,686 & 0,022 & $0,665-0,710$ & 0,500 & 0,007 & $0,495-0,505$ \\
\hline TL & 0,993 & 0,055 & $0,940-1,050$ & 1,174 & 0,019 & $1,169-1,191$ & 0,450 & 0,014 & $0,440-0,460$ \\
\hline
\end{tabular}




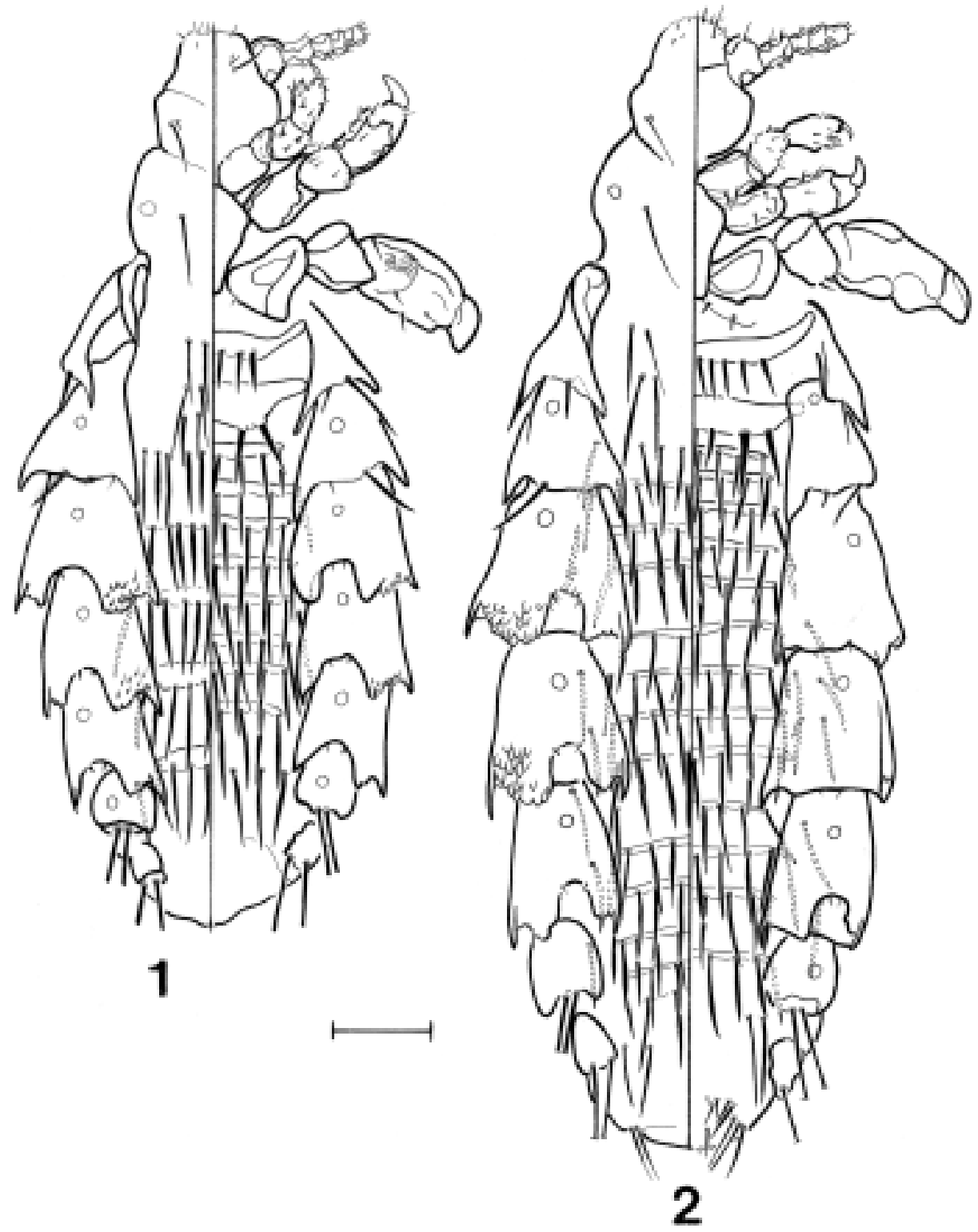

Figs. 1, 2. Hoplopleura massoiai sp. nov.: 1, macho, dorsal y ventral; 2, hembra, dorsal y ventral. Escala: $0,1 \mathrm{~mm}$. 
y borde apical redondeado; pseudopene bien desarrollado, sin dentículo alguno, y su tercio apical pronunciado terminando en una punta sagitada, la que apenas sobrepasa el nivel de los parámeros (fig. 5).

Hembra (figs. 2, 3). Características generales similares al macho, gonapofisis con escasa setación; medidas corporales (tab. I).

Primer estadio ninfal (fig. 6). Seta cefálica dorsal principal bien desarrollada, dorsal accesoria conspicua y ventral principal corta y delgada, abdomen con lóbulo anal marcado y bifurcado, llevando un par de macroquetas subterminales a cada lado; medidas (tab. I).

Huevo. Silueta elipsoidal, fijado al pelo con escasa espumalina, sin que la misma se extienda lateralmente a la base de huevo (fig. 7), tipo de ornamentación del ánfora: escamosa, apenas impresa (fig. 9); tipo de cámara aerífera: ampulácea, relación entre las cámaras aeríferas: aisladas (figs. 8, 10). Dimensiones: largo total, $495 \mu \mathrm{m}$; ancho máximo, $283 \mu \mathrm{m}$; ancho cámara aerífera, $12,9 \mu \mathrm{m}$.
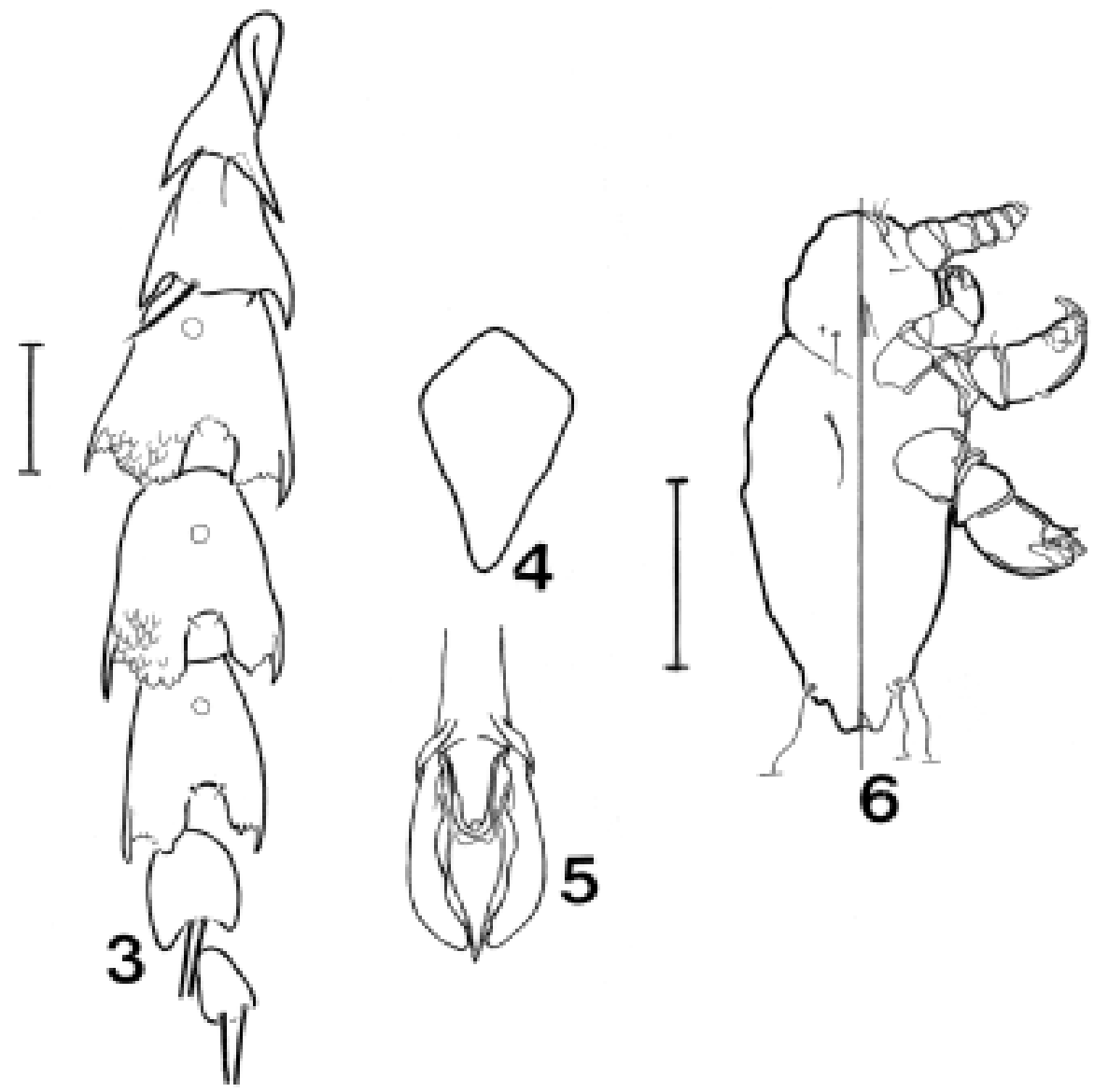

Figs. 3-6. Hoplopleura massoiai sp. nov.: 3, placas paratergales de la hembra; 4, placa torácica esternal; 5, genitales masculinos; 6, ninfa I, dorsal y ventral. Escalas: $0,1 \mathrm{~mm}$. 
Sitios de postura en el hospedador. En tres ejemplares de Bibimys torresi, B. chacoensis y B. labiosus, todos estaban parasitados, aunque en baja densidad, por Hoplopleura massoiai. Uno de los tres individuos solo portando huevos y los otros dos con escaso número de piojos, muestran sitios de postura semejantes, siempre en los flancos laterales del tórax y abdomen, mostrando mayor concentración de puestas en la región caudal.

Hospedador tipo. Bibimys torresi (Rodentia, Muridae, Sigmodontinae).

Localidad tipo. Canal 6, Estación Experimental del INTA del Delta del Paraná, Partido de Campana, Buenos Aires, Argentina.

Otros hospedadores. Bibimys chacoensis, Paso Momo, Puerto Las Palmas, Departamento Bermejo, Chaco, Argentina; B. labiosus, Departamento San Ignacio, Misiones, Argentina.

Material tipo. Holotipo ơ, alotipo $\mathcal{C}, 1$ ninfa I, ex Bibimys torresi, 09.IV.1999, D. del C. Castro col. (MLP 2899); parátipos: 2 8,3 , 1 ninfa I, ex B. chacoensis y B. labiosus, igual fecha y colector (MLP 2900).

Etimología. Hoplopleura massoiai se constituye en un homenaje a la memoria de un gran mastozoólogo argentino Sr. Elio Massoia.

Diagnosis. Hoplopleura massoiai es semejante a $H$. scapteromydis de la que se diferencia por la forma de los bordes laterales y dimensiones de la placa torácica, por la


Figs.7-10. Huevo de Hoplopleura massoiai sp. nov.: 7, general; 8, opérculo con las cámaras aeríferas y parte adyacente del ánfora; 9, detalle de la ornamentación del ánfora; 10, detalle de una cámara aerífera con la ubicación de la micrópila (a, ánfora; ach, cámara aerífera; e, espumalina; ich, cámara aerífera interna; m, micrópila; op, opérculo). Escalas: fig. 7, $100 \mu \mathrm{m}$; fig 8, 50 $\mu \mathrm{m}$; figs. 9, 10, $10 \mu \mathrm{m}$. 
presencia de lóbulos aguzados y marcada escamación terminal en las placas IV-VI, tanto en machos como en hembras; genitales masculinos con pseudopene liso, sin dentículos, y su tercio apical terminado en punta sagitada y la diferencia en número y estructura de la quetotaxia en la gonapófisis de la hembra.

Discusión. El género Hoplopleura comprende con la aquí descripta H. massoiai un total de 30 especies parásitas de roedores de Argentina. Esta nueva especie presenta características que determinan su inclusión en el grupo H. aitkeni, por la estructura de las placas paratergales y placa torácica esternal. El género Bibimys está representado en Argentina por tres especies, dos de ellas de distribución restringida y muy puntual: $B$. chacoensis, solo conocida de su localidad tipo, Puerto Las Palmas, provincia del Chaco y $B$. torresi, restringida al nordeste de la provincia de Buenos Aires y sur de Entre Ríos, Argentina; la tercera, B. labiosus, se la encuentra en por lo menos ocho localidades de la provincia de Misiones, Argentina (PARDIÑAS, 1996). Se destaca que las dos primeras son exclusivamente argentinas.

Hoplopleura massoia infesta tres especies de Bibimys, no evidenciando simpatrias en razón de su actual distribución. Aparentemente este género estaría vinculado con otros Scapteromyini, evidenciando desde el punto de vista morfológico una situación intermedia entre Scapteromys Waterhouse, 1837 y Kunsia Hershkovitz, 1966 (PARDIÑAS, 1996). La especie ahora descripta, se constituye en la primera cita de un Phthiraptera sobre este género de roedor Sigmodontinae, evidenciando parasitológicamente, una vinculación con las especies de Hoplopleura parásitas de akodontinos sudamericanos.

Agradecimientos. A Yolanda Davis y Dr. Julio Contreras del Museo Bernardino Rivadavia, Buenos Aires, Argentina, por permitirnos examinar los ejemplares de Bibimys de los cuales se extrajo el material de estudio. A la Sra. Maria Cristina Estivariz por los dibujos realizados y al Sr. Sergio Díaz por el apoyo técnico brindado.

\section{REFERENCIAS BIBLIOGRÁFICAS}

Castro, D. del C. \& Cicchino, A. C. 1978. Contribución al conocimiento de los malófagos argentinos III. Revta Soc. ent. argent., Buenos Aires, 37:77-83.

Castro, D. del C.; Cicchino, A. C. \& Villalobos, L. C. DE 1991. A comparative study of the external architecture of the eggs of some neotropical species of the genus Hoplopleura Enderlein, 1904 (Phthiraptera, Anoplura). Revta bras. Ent., São Paulo, 35(4):663-669.

Castro, D. del C.; Cicchino, A. et al. 1996. Una nueva especie del género Hoplopleura Enderlein, 1904 (Phthiraptera, Hoplopleuridae), parásita de Chelemys macronix vestitus (Thomas, 1903) (Rodentia, Cricetidae). Revta bras. Ent., São Paulo, 40(2):159-164.

Castro, D. del C.; Gonzalez, A. \& Cicchino, A. 1995. Una nueva especie del genero Hoplopleura Enderlein, 1904 (Phthiraptera, Hoplopleuridae), ectoparásita de Neotomys ebriosus vulturnus Thomas, 1921 (Rodentia, Cricetidae). Revta bras. Ent., São Paulo, 39(4):839-848.

Johnson, P. T. 1972. Sucking lice of venezuelan rodents, with remarks on related species (Anoplura). Brigh. Young Univ. Scie. Bull., Biol. Series, Utah, 17:1-62.

Kim, K. C. \& Ludwig, H. W. 1978. The family classification of the Anoplura. Syst. ento., Oxford, 3:249-284.

Pardiñas, U. F. J. 1996. El registro fósil de Bibimys Massoia, 1979 (Rodentia) en la Argentina. Consideraciones sobre los Scapteromyini (Cricetidae, Sigmodontinae) y su distribución durante el Plioceno-Holoceno en la región pampeana. Mastozool. Neotrop., Mendoza, 3(1):15-38.

Redford, K. H. \& Eisenberg, J. F. 1992. Mammals of the neotropics: The Southern Cone. Chicago, University of Chicago. 430p.

Recebido em 17.09.2002; aceito em 07.02.2003. 